\title{
Marcadores biológicos en alcohólicos abstinentes
}

\author{
Betancourt Pulsan, A.*; Cuevas Badenes, J.**; Terrado Quevedo, S.***; Valls Alvarez, M.**** \\ * Medico. Master en Drogodependencias. Facultad de Ciencias Medicas. Guantanamo. Cuba \\ * Medico. Master en Drogodependencias. Servicio de Alcoholismo. Diputacion de Valencia. \\ *** Medico. Bioquimica. Facultad de Ciencias Medicas. Guantanamo. Cuba. \\ **** Bioquimica. Profesora Auxiliar. Facultad de Ciencias Medicas. Guantanamo. Cuba.

\begin{abstract}
Resumen
Se realizó un estudio descriptivo con la finalidad de conocer las modificaciones de marcadores biológicos en una muestra de alcohólicos abstinentes; se utilizó la historia clínica de dichos pacientes. Las variables estudiadas fueron: edad, sexo, hematíes, VCM, GGT y sideremia. Los resultados destacan la superior especificidad de la sideremia con relación al VCM y GGT, lo cual refuerza el criterio del importante papel de hierro en la fisiopatología de la enfermedad alcohólica. La normalidad de los marcadores estudiados corroboró la utilidad de los mismos no sólo en el diagnóstico del alcoholismo sino en la certeza de abstinencia del paciente alcohólico.
\end{abstract}

Palabras claves: alcohólico abstinente, marcadores biológicos, sideremia.

\section{INTRODUCCIÓN}

E n 1948 la Organización Mundial de la Salud reconoció el alcoholismo como enfermedad y fue incluida en la Clasificación Internacional de Enfermedades, considerándose como población de riesgo ante el alcoholismo aquella que consume más de 7580 gramos de alcohol puro al día.

Los últimos años acusan un incremento del $30 \%$ en el consumo de alcohol en los países desarrollados (González Mateo;Buitrago, 1993); si bien el consumo de etanol y los problemas relacionados con el mismo se han empezado a estabilizar en los países europeos, en otros países del mundo en vías de desarrollo, hay un claro aumento del consumo y de los problemas asociados.

De hecho se está confirmando en todo el mundo una modificación en los hábitos de consumo: el con-

\section{Summary}

A descritptive study was made in order to know the modifications of biological

Markers in a sample of abstinent alcoholics, with the clinical chart of these patients, taking into account the age, sex, red cells, VCM, GGT and sideremy. The results enhanced the high specifity of sideremy in relation to VCM and GGT which reinforce the criterion of the important role of the iron in the physiopathology of this alcoholic illness. Normality of the patterns studied strengthen their utility not only in the diagnosis of alcoholism but also in the certainty in the abstinence of the alcoholic patient.

Key words: abstinent alcoholic patient, biological markers, sideremy.

sumo aumenta cuando aumenta el nivel cultural y los ingresos económicos (Rodés; Bach;Bach, 1990).

España es uno de los principales consumidores de alcohol en el mundo $(19,21)$, se sitúa detrás de Francia $(20,81)$, y de Luxemburgo $(20,1)$.Un $10 \%$ de la población española presenta un consumo de alto riesgo de bebidas. El grupo de población más afectado por los problemas del alcohol, es de 45-64 años de edad. Este consumo excesivo afecta mucho mas a los varones $(18,1 \%)$ que a las mujeres españolas $(2 \%)$. EI segundo grupo mas perjudicado es el de los jóvenes de 16-24 años (Plan Nacional, 1991).

Esta realidad hace que el diagnóstico del alcoholismo sea muchas veces difícil debido que el paciente minimiza o esconde la ingesta de alcohol, o no se considera un enfermo o no relaciona su sintomatología con el consumo de alcohol (Casas; Gutiérrez; San, 1994). 
Para el diagnóstico del alcoholismo se utilizan diversos métodos, entre los que se destacan el interrogatorio del paciente y sus allegados, los cuestionarios mas o menos complejos y una serie de pruebas de laboratorio a las que se les ha denominado de manera genérica Marcadores Biológicos de Alcoholismo (Caballería, 1996; Casas; Gutiérrez; San, 1994, Rodés; Urbano; Bach, 1990).

Las características que debe poseer un prueba de laboratorio para ser considerada un buen marcador biológico de alcoholismo es que tenga una alta sensibilidad y especificidad, que pueda realizarse rutinariamente en un laboratorio de tipo medio y que sirva tanto para el diagnóstico del alcoholismo como para el control de la abstinencia de los pacientes sometidos a tratamiento de deshabituación (Casas; Gutiérrez; San, 1994, Rodees; Urbano; Bah, 1990).

La utilidad de los distintos marcadores varía mucho. Ello puede deberse a la heterogeneidad de las poblaciones estudiadas respecto al consumo alcohólico, al tiempo transcurrido entre la última ingesta y la realización de las pruebas analíticas y a las diferencias relacionadas con la presencia y gravedad de una posible hepatopatía asociada a partir de graves cambios metabólicos provocados en la ingestión crónica (Hernández; Sánchez; Barbaños, 1995; Casas; Gutiérrez; San, 1994; González, 1994).

Por otro lado la mayoría de estas pruebas de laboratorio presentan la dificultad de su inespecificidad y ninguna por si misma o en combinación puede dar un diagnóstico inequívoco de alcoholismo, puesto que pueden estar alteradas por:

-Múltiples enfermedades, además de las hepáticas, sanguíneas, cardíacas y renales.

-Ingesta de fármacos y concretamente aquellos que provocan una inducción enzimática, como barbitúricos, anticonvulsivantes y hormonas tiroideas.

-Otros factores como la edad, el consumo de taba$\mathrm{co}$, intervenciones quirúrgicas o radioterapia.

A pesar de esto no deben menospreciarse los resultados anormales de estas pruebas (Rodrigo, 1993; Soler-Insa; Freixa; Reina, 1998).

Entre los marcadores biológicos que ayudan a corroborar la sospecha clínica de alcoholismo y a controlar la abstinencia se destacan: volumen corpuscular medio de los hematíes (VCM), hematíes, gamma glutamil transpeptidasa (GGT), y sideremia (Caballería, 1995; Díaz; Gual, 1994; Shüller, 1991: Rodés et al, 1990).

Volumen Corpuscular Medio de los Hematíes (VCM):

El aumento de VCM es un hallazgo común en los alcohólicos. La relación entre macrocitosis y el alcoholismo ha sido confirmado por numerosos estudios.
El porcentaje de alcohólicos con una elevación del VCM varía de unas series a otras, oscilando entre un 31 y el $96 \%$.

La macrocitosis en los alcohólicos se debe a un efecto tóxico directo del alcohol. La existencia de una enfermedad hepática también contribuye a la macrocitosis de los alcohólicos. La frecuencia e intensidad de la misma es mayor en los alcohólicos con una hepatopatía asociada que en aquellos sin hepatopatías.

La especificidad del VCM es alta, especialmente en los pacientes ambulatorios, en los que otras causas de macrocitosis son muy pocos frecuentes.

EI VCM disminuye lentamente a los 3-4 meses, y se vuelve a elevar si se reinicia la ingestión de alcohol.

EI VCM también puede elevarse cuando existe déficit de ácido fólico, vitamina B12, en la reticulocitosis, por la ingestión de algunos medicamentos como antiepilépticos, por el tabaco, con la edad y en la menopausia (Caballería, 1996_Casas et al, 1994_Rodés et al, 1990).

\section{GGT}

La gamma glutamil transpeptidasa (GGT) es probablemente el marcador de alcoholismo más utilizado en la actualidad por su elevada sensilibidad y por la facilidad de realización. En el hígado la GGT se halla en las membranas de los hepatocitos y en los microsomas de las células, especialmente en las situadas alrededor de los conductillos biliares.

La GGT también se eleva en diversas circunstancias como en las colestasis, en enfermedades hepáticas de cualquier etiología y después de administración de fármacos inductores del sistema microsomal de los hepatocitos.

La GGT puede elevarse además en múltiples procesos patológicos extrahepáticos, como obesidad, diabetes, pancreatitis, insuficiencia cardíaca y traumatismos graves. Por todo ello, la alta sensibilidad de la GGT se acompaña de una especificidad relativamente baja.

La ingestión aguda de alcohol no modifica los valores séricos de GGT ni en voluntarios sanos ni en hepatópatas. Por el contrario, una elevación de la GGT se observa en el 35-85 \% de los alcohólicos crónicos.

En muchos casos las cifras de GGT son 2-3 veces superiores al valor normal, aunque no es raro que aumenten hasta 10-20 veces, dependiendo de la intensidad del consumo de alcohol y de la existencia o no de una enfermedad hepática.

La abstinencia se acompaña de una reducción progresiva de los valores séricos de GGT, se alcanzan cifras normales al cabo de seis a ocho semanas, siempre que no exista una enfermedad hepática, en cuyo caso los valores pueden aparecer discretamente elevados. 
Las cifras de GGT aumentan de nuevo al reanudarse el consumo de alcohol, por lo que su determinación periódica es útil para el control de la abstinencia de los pacientes sometidos a tratamiento de deshabituación (Halex; Stuart; Yodosfsky; Talbott. 1996; Casas et al, 1994; Miller; Gold, 1993; Rodés et al, 1990).

El mecanismo de elevación de la GGT en los alcohólicos no es conocido y aunque se ha considerado que podría estar en relación con un proceso de inducción enzimática es posible que incluso en los alcohólicos sin enfermedad hepática la elevación de la GGT traduzca un cierto grado de disfunción del hepatocito.

\section{SIDEREMIA}

El etanol favorece la absorción del hierro y ésta se acelera si existe concomitante mente una pancreatitis crónica alcohólica (Shüller, 1991). Esta pancreatitis crónica aumentaría a su vez la absorción del hierro.

La absorción del hierro se favorece por la ingestión de alcohol porque éste estimula la secreción gástrica, por la insuficiencia pancreática que cursa con disminución en la excreción del bicarbonato y del polipéptido ligador del hierro, inhibidor de su absorción (cuevas, 1995).

Hay autores que observan descensos moderados, no significativos, de la sideremia y la transferrina en los alcohólicos más graves, es decir, los que hacen ingestas superiores a 200 gr. diarios de etanol (Conde, 1993; Pares, 1990); Para otros autores la sideremia se encuentra en valores normales no estando elevados los depósitos férricos (Shüller, 1991); En otros estudios se han encontrado niveles de sideremia elevados en el 40\% de los mismos (Cuevas; Torres; Rubio, 1994).

Por otra parte se han descrito niveles elevados de ferritina en el $67 \%$ de los alcohólicos crónicos sin lesiones hepáticas (Rodés et al, 1990). No obstante, el hierro es un oligoelemento de gran trascendencia en la clínica del paciente alcohólico.

Teniendo en cuenta que entre los marcadores clásicos de alcoholismo están el VCM, la GGT y la Sideremia y que éstos por su fácil realización y sensibilidad son útiles para el diagnóstico del alcoholismo pero también para el control de la abstinencia, durante el tratamiento de deshabituación, se realiza el presente estudio orientado a explorar las modificaciones de algunos de estos marcadores biológicos en una muestra de alcohólicos después de 6 meses y de un año sin consumo etílico.

\section{MATERIAL Y METODO}

Se realizó un estudio descriptivo de pacientes alcohólicos, atendidos en el Centro Ambulatorio de aten- ción al Alcoholismo de la Asociación Valenciana de Ex alcohólicos (AVEX), de la ciudad de Valencia, durante el período de octubre de 1994 a abril de 1996. Fueron seleccionados todos aquellos enfermos que tenían entre 6 meses y un año sin consumir bebidas alcohólicas, se excluyeron todos los que tuvieron al menos una recaída en el período señalado, no cumplían el tiempo mínimo de abstinencia o no había constancia de los exámenes de laboratorio efectuados; la muestra final quedó conformada por 72 pacientes $(n=72)$.

La información fue recogida de las historias clínicas de los pacientes con diagnóstico de dependencia alcohólica según los criterios del DSM-IV (DSM-IV, 1995).

De cada una de las historias clínicas se seleccionaron las variables siguientes: hematíes, volumen corpuscular medio de los hematíes, hierro y gamma glutamil transpeptidasa. Se comprobó que estas variables se encontraban alteradas en los pacientes alcohólicos antes de iniciar el tratamiento de deshabituación.

Fueron considerados rangos de valores normales para cada prueba de laboratorio, los referenciados por los laboratorios del Servicio Valenciano de Salud. Se realizó la conversión a las normas de este servicio en los pocos casos (tres) en que dichas pruebas se efectuaron en otros laboratorios.

Los datos se presentan en tanto por ciento con media y desviación estándar.

\section{RESULTADOS}

\section{Edad}

La edad media fue de 41, 9 años, con una desviación típica de 9, 14. Los rangos de edades de 25-34 y de 35-44 años fueron los que predominaron en el $50 \%$ de la muestra (Fig. 1).

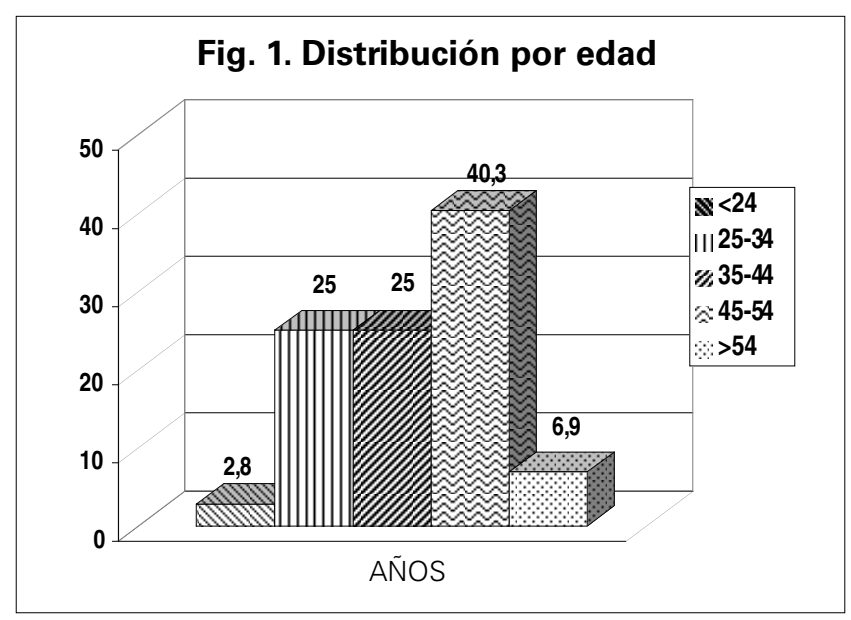




\section{Sexo}

Predominó la población masculina en el 80,6\% del estudio (Fig. 2). La proporción hombre/mujer fue de 4:1.

\section{Fig. 2. Distribución por sexo}

El análisis de la muestra atendiendo a la edad y el sexo permitió destacar que ninguna mujer se encontró en el rango correspondiente a menor de 24 años; el $44,8 \%$ de los varones estuvo en el rango de $45-54$ años frente al $50 \%$ de las mujeres.(Fig. 3).

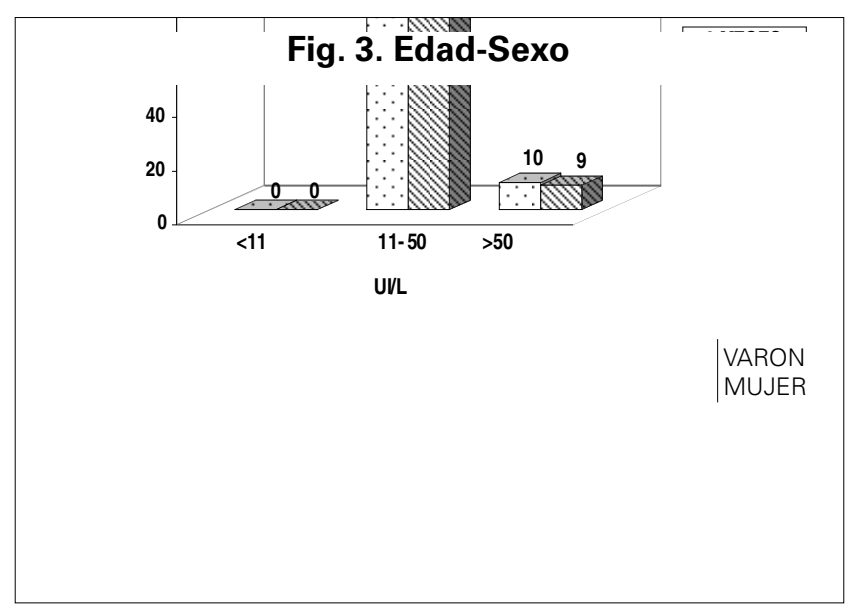

\section{Hematíes}

En la muestra estudiada las medias fueron de 4.723.650, con una desviación típica de 386.232 y 4.822.666, con una desviación de 411.129 para los 6 meses y un año respectivamente. Resultó relevante que el $100 \%$ de los casos estudiados tenía las cifras de hematíes dentro de los rangos considerados como normales al año de abstinencia (Fig.4).

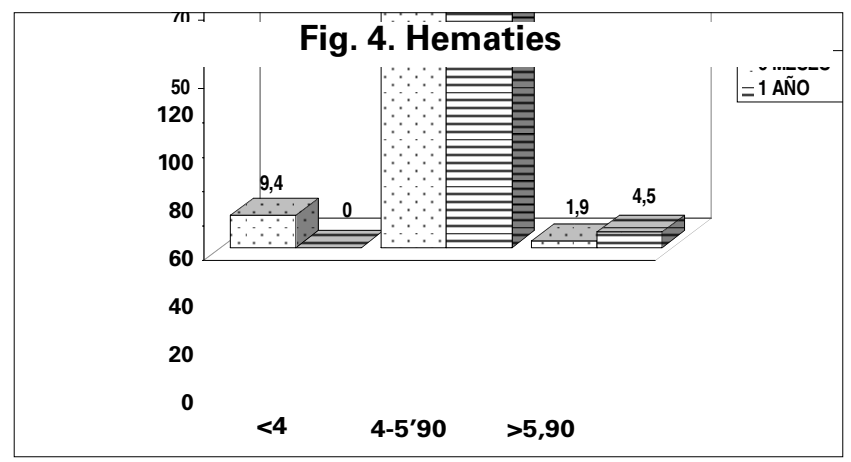

VCM

El VCM en la muestra estudiada se comportó con una media de $92.13 \mathrm{fl}$, y una desviación standard de 5.29 a los 6 meses. Se encontró que el 92,0\% de la muestra tenía los valores dentro de los límites normales.

La media al año de abstinencia fue de $90.93 \mathrm{fl}$ y la desviación de 5.53.El 93.4\% de la muestra presentó cifras normales (Fig. 5).

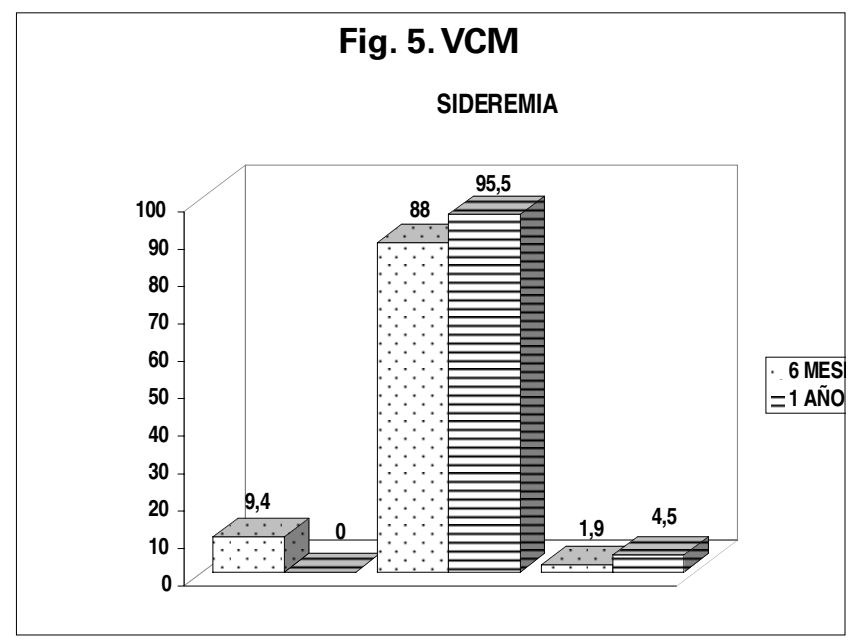

\section{Sideremia}

La media de sideremia en los casos estudiados con 6 meses de abstinencia fue de 85.40 UG/DL con una desviación de 36.81. El 88.7\% de la muestra reflejó cifras dentro de los parámetros normales.

Para los de un año se encontró una media de 90.23 UG/DL con una desviación típica de 24.39. EI $95.5 \%$ de la muestra estudiada presentó valores normales. (Fig. 6).

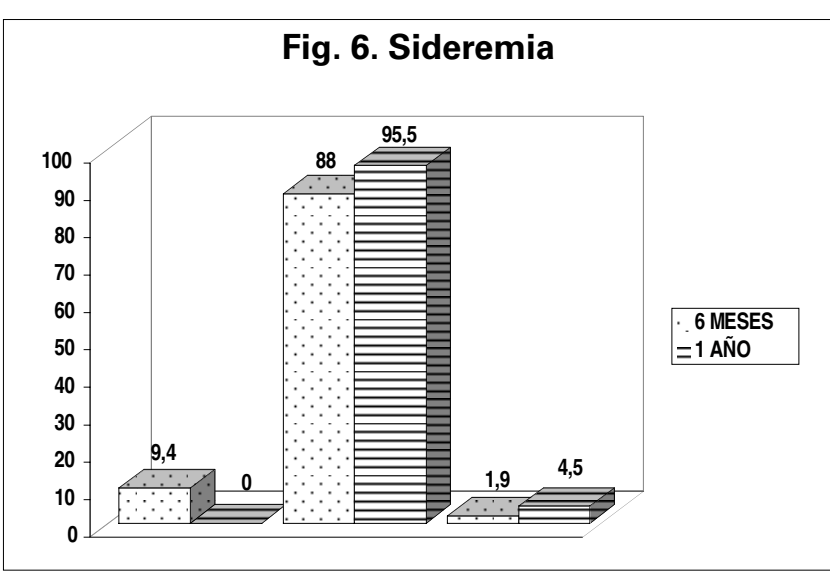

\section{GGT}

La Gamma Glutamil Transpeptidasa Sérica tuvo el siguiente comportamiento: a los 6 meses la media 
resultó de $31.17 \mathrm{UI} / \mathrm{L}$, con una desviación de 44.60. El $90 \%$ de los casos estudiados presentó valores normales.

Al año de abstinencia la media fue de 23.91 con la desviación de 21.74 y con el $91 \%$ de los sujetos de la muestra con cifras normales (Fig. 7).

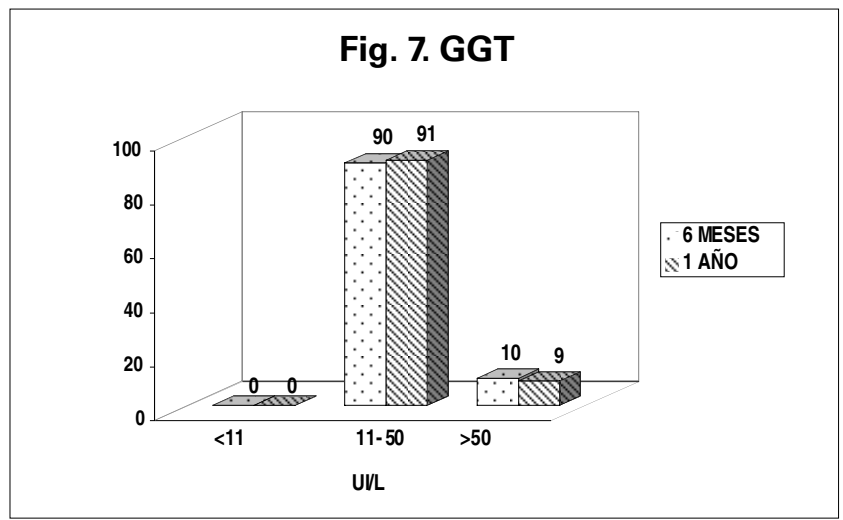

\section{DISCUSION}

\section{Edad}

El resultado encontrado por nosotros ha sido similar a lo referido en numerosos estudios, así autores como Mateo y colaboradores encuentran en alcohólicos primarios ambulatorios una edad promedio de 43.5 (Mateo; Gumá; González, 1995), Cuevas señala una edad media de 41.6 en alcohólicos ingresados en una Unidad de Desintoxicación Hospitalaria (Cuevas et al, 1994), González y colaboradores en 200 alcohólicos estudiados reflejan 42 años como la edad predominante (González; Mateo; Buitrago, 1993), resultados similares obtienen Cortés (Cortés; Pascual; Torres, 1994), González (González; Garriga; Ceiro et al, 1991) y otros autores como Alonso (Alonso; Martínez; Morales, 1994) y Rodríguez (Rodríguez; Montané; Crespo et al, 1993).

El alcoholismo es un problema que se manifiesta en las décadas de la vida en que se establecen compromisos en las relaciones íntimas, carreras, nexos en comunidades importantes para la vida (Sagava; Pak, 1991), lo que obliga a un esfuerzo mancomunado de toda la sociedad y adultos jóvenes en particular, para preservar la salud y enfrentar esta enfermedad como una preocupación común.

\section{Sexo}

Todos los estudios epidemiológicos realizados tanto en España como en EEUU dan un predominio de la enfermedad alcohólica en hombres, en proporciones que van desde 5/1 hasta7/1, muy aproximadas a la encontrada en este trabajo (DSM IV, 1995; Cuevas et al, 1994; Cortés et al, 1994; González et al, 1993; Miller y Bold, 1993). Durante la presente década se observa una tendencia hacia la reducción de las diferencias entre sexos, sobre todo en la población más joven (Secades, 1996; Miller y Gold, 1993; Centro Valenciano de Doc., 1993, Plan Nacional sobre Drogodependecias, 1991).

\section{Hematíes}

En nuestro estudio resultó relevante que el 100\% de los casos estudiados tenían cifras las cifras de hematíes dentro de los rangos considerados normales al año de abstinencia.

Autores como Cuevas y colaboradores en un estudio realizado 170 alcohólicos sometidos a tratamiento de deshabituación encuentran que el $34.1 \%$ de los pacientes tenían una tasa inferior a 4.1 millones de hematíes por cc (Cuevas;Torres Rubio, 1994).

En el transcurso del alcoholismo crónico, suele encontrarse anemia acompañada de trastornos nutritivos con déficit de ácido fólico y de hierro.(Valvuena, 1993).

\section{VCM}

Numerosos estudios evidencian alteraciones en el VCM en alcohólicos, así Cuevas y colaboradores encuentran en 170 alcohólicos en tratamiento que el $56.5 \%$ tienen el VCM superior a 99 fl. (Cuevas; Torres; Rubio, 1994);González y colaboradores en una muestra con $n=200$ encuentran que el $51 \%$ tienen el VCM elevado(González, Garriga, Ceiro et al, 1991).

En nuestro estudio a los seis meses de abstinencia el 92, $0 \%$ de los pacientes presentó cifras normales y al año de abstinencia el 93, 4 \% presentó cifras normales.

EI VCM es uno de los marcadores habituales no solo el diagnóstico del alcoholismo, sino también en el control de la abstinencia en los alcohólicos. El estudio realizado corrobora una vez más la utilidad de ese parámetro.

\section{Sideremia}

Los reportes del efecto del alcohol en el metabolismo del hierro no son concluyentes (Velasco, 1998).

Cuevas y colaboradores encuentran que en el 40 $\%$ de los casos de su estudio la sideremia está elevada teniendo en cuenta que los casos estudiados son pacientes ingresados para su desintoxicación, resultado que conjuntamente con el obtenido en el presente trabajo avala la importancia de la determinación del hierro como marcador biológico al observarse su alteración durante el consumo y su normalización durante la abstinencia (Cuevas, Torres, Rubio, 1994). 
Los resultados en los diferentes estudios son sumamente discrepantes, algunos autores han encontrado un predominio de la sideremia elevada; mientras otros autores encontraron la sideremia disminuida; pero tal y como señala Cuevas en su estudio, el hierro es un oligoelemento de una gran trascendencia en la clínica del paciente alcohólico (Cuevas, 1995). Coincidiendo con el criterio de este autor consideramos que se hace imprescindible las determinación del hierro en cualquier batería analítica que se le solicite a un consumidor de etanol teniendo en cuenta que aún existen profesionales que continúan utilizando protocolos de tratamiento para enfermos alcohólicos en los que se prescriben suplementos férricos que favorecen la sobrecarga de este mineral y por ende pueden agravar los cuadros clínicos. Por otro lado el aumento de la sideremia puede tener un rol protagónico en la fisiopatología de la Enfermedad Alcohólica, al olvidar que el etanol favorece la absorción de hierro.

\section{GGT}

Cuevas y colaboradores describen en su estudio, que el 53, 5\% de su muestra tiene cifras de GGT elevadas (Cuevas, Torres, Rubio, 1994). En otros estudios se describen cifras de GGT también elevadas(Hales, Yudofsky, Talbott, 1996; Miller, Gold, 1993).

El predominio de cifras dentro de los rangos de normalidad del presente estudio contribuyen a corroborar que la elevada sensibildad de la GGT para detectar consumo excesivo de alcohol también es un indicador útil para la evaluación de la abstinencia.

\section{CONCLUSIONES}

-El alcohólico abstinente del presente trabajo, es un adulto joven con edad que oscila entre la tercera y la cuarta década de la vida, continúa siendo predominantemente del sexo masculino aunque la relación hombre/mujer se estrecha cada vez más.

-Al año y a los seis meses de abstinencia la mayoría de los pacientes presentó valores normales de VCM y de sideremia.

-Los resultados destacados de normalidad en los marcadores biológicos estudiados corroboran la utilidad de los mismos, en la certeza de abstinencia del alcohólico.

\section{BIBLIOGRAFIA}

Alonso, O; Martínez, F; Morales, M.(1994).Caracterización de alcoholismo en el municipio de Camagüey, Cuba, 1986-1990.Rev.Esp. Drog. 19(2):117-130.
Caballería, J y Caballería, L.(1996). Marcadores biológicos en el diagnóstico del alcoholismo. Lanol. (1157): 28-32.

Casas, M; Gutiérrez, M, Sanz, L. (1994). Psicopatología y alcoholismo. De. Neurociencias. Barcelona.

Centro Valenciano de Documentación sobre Drogodependencias (1993). Boletín de información sobre drogodependencias (14/2/93).

Conde, J.M; De la Gandora, J; Medina, M.A; Blanco, M. (1993). Transferrina y diagnóstico del alcoholismo. AN. Acad. Med. y Cir. Vall. Vol XXXI: 155-123.

Cortés, M.T; Pascual, M; Torres, M.A. (1994). Estudio de alcoholismo en un Área sanitaria de la comunidad valenciana. Adicciones. 6(1): 23-50.

Cuevas, J. (1995). Metabolismo del hierro. Su rol en la clínica del alcoholismo. Rev. Esp. Drog. 20 (4) : 307-302.

Cuevas; J; Torres, M.A. Rubio, J. (1994). Estudio descriptivo de los pacientes alcohólicos ingresados en una unidad de Desintoxicación Hospitalaria. Rev. Esp. Drog. 19 (4): 325-345.

D.S.M. IV (1995). Manual diagnóstico y estadístico de los trastornos mentales. De. España. Mason, S.A. Madrid.

Diaz, R, Gual, A. (1994). ¿Bebe Ud. alcohol en exceso?. Adicciones 6 (2): 211-229.

González, R; Mateo, A; Buitrago, J. (1993). Las motivaciones para beber: estudio en alcohólicos y bebedores sociales. Rev. Esp. Drog. 18 (2): 85-92.

González, R; Garriga, L; Ceiro, L; Reyes, J; Calzadilla, L. (1991). Los cuestionarios breves y las pruebas hematológicas inespecíficas: estudio comparativo en dependientes alcohólicos y bebedores sociales. Rev. Esp. Drog. 16 (4): 305-319.

González, R. (1994). ¿Cómo liberarse de los hábitos tóxicos? (1era parte: guía para conocer y vencer los hábitos provocados por el café y el tabaco). Rev. Esp. Drog. 18(1): 61-71.

Hales, R; Yudosfsky; S;Talbott, J. (1996). Tratado de Psiquiatría. 2da de. Ancora, S. A. Barcelona.

Hernández, A; Sánchez, M; Barbaño, M. (1995). Genética del alcoholismo crónico: identificación de marcadores biológicos de lata especificidad. Rev. Esp. Drog. 20 (4): 297-305.

Mateo, A; Gumá, E; González, R. (1995).Estudio comparativo de tres grupos de alcohólicos. Rev. Esp. Drog. 20 (1): 41-47.

Miller, N; Gold, M. (1993). Alcohol. Ed. Neurociencias. Barcelona.

Pares, A. (1990). Lesiones hepáticas inducidas por el alcohol. Clínica y Anatomía Patológica. En alcohol y enfermedad. Barcelona J. R. Proust, S. A. 141-158.

Plan Nacional sobre Drogodependencias (1991). Unidades de Desintoxicación Hospitalaria (UDH). Informe Año 1990. Ministerio de Sanidad y Consumo. Madrid.

Rodés, J; Urbano- Márquez, A; Bach; Bach, L. (1990). Alcohol y enfermedad. J. K. Proust. Ed.Barcelona.

Rodrigo, J; Escudero. A: (1993). Hígado y alcohol. Cuadernos de CVDD: $4^{0}$ Congreso del SET 5 (3): 85-89. 
Rodríguez, T; Montané, A; Crespo, R; Sierra, M: (1993).Motivaciones influyentes en la habituación alcohólica. Rev. Esp. DROG. 18(3): 173-180.

Sadava, S; Pak, A: (1991):Desarrollo psicosocial durante la tercera década de la vida y problemas alcohólicos. Estudio longitudinal avances psicología clínica latinoamericana. 9: 185-202.

Shüller; A. (1991).Alcohol y enfermedad. Eudema. Madrid.
Secades, R. (1996).Alcoholismo juvenil. De.Pirámides. Col. Ojos Solares Madrid.

Soler-Insa, P; Freixa, F; Reina, F.(1998). Trastornos por dependencia del alcohol. Conceptos actuales. Lab. De la Grange. Madrid.

Valbuena, A: (1993).Toxicomanías y alcoholismo. 2da. Ed. Científica y Técnica, S. A. Barcelona.

Velasco, R. (1998). Alcoholismo. Visión Integral Ed. Trillas. Méxic. 
